Service social

\title{
Le repérage des zones de pauvreté sur les territoires de C.L.S.C. Une méthodologie de recherche pour l'action
}

\section{Jocelyne Barnabé et Gérald Doré}

Volume 38, numéro 2-3, 1989

Les politiques sociales

URI : https://id.erudit.org/iderudit/706442ar

DOI : https://doi.org/10.7202/706442ar

Aller au sommaire du numéro

Éditeur(s)

École de service social de l'Université Laval

ISSN

1708-1734 (numérique)

Découvrir la revue

Citer cet article

Barnabé, J. \& Doré, G. (1989). Le repérage des zones de pauvreté sur les territoires de C.L.S.C. - Une méthodologie de recherche pour l'action. Service social, 38(2-3), 270-281. https://doi.org/10.7202/706442ar
Résumé de l'article

Des études récentes confirment la relation entre, d'une part, la pauvreté et, d'autre part, les maladies et les problèmes psychosociaux sur lesquels les services de première ligne des C.L.S.C. sont appelés à intervenir. Cet article propose une méthodologie facile d'application, peu coûteuse, tout en étant rigoureuse et fiable, pour le repérage des zones de pauvreté sur les territoires de C.L.S.C. 
BARNABÉ, Jocelyne, organisatrice communautaire au C.L.S.C. La Source.

DORÉ, Gérald, professeur à l'École de service social de l'Université Laval.

\section{Le repérage des zones de pauvreté sur les territoires de C.L.S.C. - Une méthodologie de recherche pour l'action}

\section{Jocelyne Barnabé Gérald Doré}

Agir comme organisateur ou organisatrice* communautaire dans un centre local de services communautaires (C.L.S.C.), c'est travailler à appuyer ou susciter l'action collective des communautés du territoire sur les situations-problèmes de leurs conditions de vie qui sont reliées à l'incidence des maladies et des tensions psychosociales auxquelles sont appelés à répondre les services de première ligne du C.L.S.C. L'exercice de cette fonction, en relation avec celles des autres intervenants et intervenantes du C.L.S.C., implique la capacité de localiser sur le territoire les agglomérations où se concentrent les populations à animer en priorité et dont les besoins sont les plus marqués en regard des services que dispense ou que pourrait éventuellement dispenser le C.L.S.C., dans une optique d'ajustement de sa mission sociale aux conditions spécifiques du territoire où elle s'exerce.

Cette philosophie d'action, qui est en soi une prise de position dans les débats actuels sur l'action communautaire dans les C.L.S.C., inclut, dans la fonction de l'organisateur ou de l'organisatrice communautaire, la tâche de réaliser des études sur le milieu, en s'adjoignant les compétences utiles, suivant les ressources disponibles. À ce titre, elle a inspiré une mini-recherche réalisée en 1986 et 1987 au C.L.S.C. La Source, à travers les multiples autres tâches 
qui échoient à une organisatrice communautaire, dans un établissement qui ne compte que deux postes d'organisation communautaire, pour un immense territoire s'étendant de Charlesbourg aux limites de la réserve faunique des Laurentides et comptant plus de 80000 habitants.

Jocelyne Barnabé a sollicité et obtenu le mandat ${ }^{1}$ de réaliser la recherche et en a assumé la direction. Gérald Doré, professeur à I'École de service social de l'Université Laval, a agi comme consultant. Une étudiante du programme de doctorat en sociologie de l'Université Laval, Anne Racine, a exécuté les travaux de la recherche. Denis Fortin, professeur à l'École de service social de l'Université Laval et Jean-Pierre Labeau, graphiste au Service des ressources pédagogiques de l'Université Laval, ont collaboré à la cartographie des résultats.

\section{Un objet de recherche lié à une priorité d'action}

La recherche avait pour objet le repérage des zones de pauvreté sur le territoire du C.L.S.C. et l'étude de leurs caractéristiques. Le choix de ce thème découle directement de la philosophie d'action énoncée plus haut. Comme tous les établissements de sa catégorie, le C.L.S.C. La Source a pour mission de servir l'ensemble de la population de son territoire, dans une optique essentielle d'universalité des services qui n'exclut pas, cependant, une attention prioritaire à ce qu'il est convenu d'appeler dans le jargon institutionnel les "clientèles à risques ». Or, étudier la distribution géographique de la pauvreté sur le territoire d'un C.L.S.C., c'est contribuer de manière significative à la localisation des dites clientèles.

Des études récentes ont en effet confirmé ce qui était déjà connu depuis longtemps (Hollingshead et Redlich, 1958; Schlesinger, 1965; Laplante, 1966), à savoir la relation étroite entre pauvreté d'une part, et maladie ou problèmes psychosociaux d'autre part. Le rapport de l'enquête Santé Québec 1987 (Émond, 1988) révèle que :

Le revenu est associé à la fréquence et à l'impact des événements de vie. Les groupes les plus pauvres sont à la fois deux fois plus susceptibles de connaître des événements stressants et de les vivre de façon plus pénible que ne le sont les personnes des catégories plus élevées (p. 80, 81).

Les personnes plus défavorisées économiquement ont plus de problèmes de santé que les plus riches, particulièrement au chapitre des affections les plus sévères, tels les troubles mentaux et les maladies cardiaques (p. 105).

L'ensemble de la population démunie déclare deux fois plus souvent une limitation dans ses rôles sociaux que la population ayant des revenus moyens ou élevés (p. 196). 
De son côté, Chamberland (1987) démontre, à partir d'une revue de la littérature et d'une recherche qu'elle a effectuée à Montréal, " l'impact des conditions d'appauvrissement économique et social des familles sur l'ampleur du phénomène des mauvais traitements envers les enfants "(Chamberland, $1987: 274)$. Elle rappelle en outre les conclusions de Egbuonu et Starfield (1982), de même que celles de Nersesian et al. (1985), à l'effet que "les caractéristiques généralement reliées à de plus grands risques de mauvais traitements sont également associées à la pauvreté, comme la présence de bébés de petits poids, prématurés ou difficiles à soigner, ou encore d'enfants handicapés"(Chamberland, $1987: 276$ ). À cet égard, il semble bien que seulement " un coin de la couverte " ait été soulevé, si on en juge par cette fuite journalistique du début de 1989, au sujet d'une étude du département de santé communautaire de l'Hôpital Saint-Luc de Montréal, remise au ministère de la Santé et des Services sociaux en 1987, mais non rendue publique par lui (Bellefeuille, 1989). On y rapporte notamment que le Québec se place sur un pied d'égalité avec les pays du Tiers-Monde en ce qui a trait au taux d'insuffisance de poids à la naissance.

Or, pour ne s'en tenir qu'à ce seul exemple, des intervenants et intervenantes de C.L.S.C. impliqués dans les programmes de périnatalité ont déjà constaté leur difficulté à rejoindre les femmes pauvres enceintes par les approches conventionnelles, censées s'adresser à l'ensemble des femmes enceintes d'un territoire (Duhaime, 1987 : $216,223)$. Cette constatation laisse perplexe et incite à s'interroger sur les programmes dirigés vers d'autres "populations-cibles».

La pauvreté est une dimension déterminante de la réalité sociale qui confronte les C.L.S.C. La pertinence d'étudier la distribution géographique de la pauvreté sur les territoires de C.L.S.C. ne fait pas de doute, mais pour donner suite pratiquement à cette exigence du rapport au milieu, encore faut-il disposer de moyens qui soient compatibles avec le contexte du C.L.S.C., qui en est un d'action et non prioritairement de recherche.

\section{Une méthodologie rigoureuse mais simple, peu coûteuse et généralisable}

Il existe des méthodes complexes et sophistiquées pour mesurer la distribution de la pauvreté sur un territoire. Celle utilisée, par exemple, par Côté, Doré et Dussault, en 1971, sur le territoire de la ville de Québec, appartient à cette catégorie. Elle combine de multiples indicateurs (scolarité, revenu, travail, charges familiales, logement) qu'elle intègre dans une mesure de désavantage socioéconomique en recourant aux techniques de construction d'échelle. 
L'écologie factorielle (Mayer-Renaud, 1988) est une autre de ces méthodes. Celles-ci présentent un intérêt indéniable, mais elles requièrent une compétence avancée dans les techniques quantitatives de recherche et une lourde procédure de saisie et de traitement des données. Leur complexité les rend de plus difficilement explicables à des non-initiés.

La méthode que nous avons choisie s'avère au contraire très simple, tout en étant rigoureuse et tout à fait défendable sur lé plan scientifique. Elle est facilement explicable, aussi bien aux gens du milieu qu'elle concerne qu'aux membres du personnel de l'établissement; elle coûte peu cher d'application, en temps comme en argent ${ }^{2}$, ce qui ne peut manquer de lui conférer un attrait supplémentaire dans l'état actuel des choses. Les qualités qui précèdent la rendent, par conséquent, généralisable à tous les C.L.S.C. qui partagent notre intérêt.

Cette méthode ne repose que sur un seul indicateur socioéconomique dont le rapport à la pauvreté est clair pour tous : le revenu. Elle réfère de plus à un concept maintenant largement diffusé dans le grand public, celui de "seuil de pauvreté ". Toute la stratégie de la recherche consiste à étudier la distribution des ménages ${ }^{3}$ vivant sous le seuil de la pauvreté, sur le territoire du C.L.S.C., et à observer leurs caractéristiques. Elle se réalise exclusivement à partir des données du recensement et des services offerts par Statistique Canada et par le Bureau de la statistique du Québec.

II existe plusieurs définitions des seuils de pauvreté au Canada : celle du Comité du sénat sur la pauvreté, celle du Conseil canadien de développement social (organisme non gouvernemental) et celle de Statistique Canada, reprise par le Conseil national du Bien-être social (organisme gouvernemental), dans une publication annuelle qui donne les seuils pour l'année en cours, en les comparant à ceux des années récentes. Les seuils du Comité du sénat et du Conseil canadien de développement social sont plus élevés que ceux de Statistique Canada et, par conséquent, retiennent comme " pauvres » plus de ménages que ceux de Statistique Canada. Nous avons néanmoins opté pour ces derniers, non seulement à cause de la plus grande accessibilité des informations (mise à jour annuelle dans une publication largement diffusée), mais aussi à cause du caractère sévère (conservateur) de cette définition des seuils. On ne peut en aucune façon être taxé d'exagérer la pauvreté sur un territoire quand on utilise ces seuils.

Selon la définition de Statistique Canada, les familles sous le seuil de pauvreté sont celles qui consacrent $20 \%$ de plus que la moyenne à la nourriture, aux vêtements et au logement. Comme les enquêtes réalisées par Statistique Canada sur les dépenses des familles ont 
révélé que cette moyenne était de $38,5 \%$, les seuils de faible revenu sont fixés à des niveaux où on consacre en moyenne $58,5 \%$ du revenu aux trois items précités (CNBES, $1988: 1)^{4}$. Une fois cette norme établie, Statistique Canada trouve dans chaque catégorie (personne seule, famille de deux personnes, etc.) le revenu moyen de ceux qui consacrent 58,5\% aux besoins de base (CNBES, 1988 : 2-3). Cette opération n'est pas effectuée pour l'ensemble du Canada, mais bien plutôt par catégorie de milieu de résidence : les régions métropolitaines comptant un demi-million de résidents ou plus (dont Québec), les grandes villes (100 000 à 499 999), etc.

Les seuils de pauvreté que nous avons utilisés s'appliquaient à la région métropolitaine de Québec, où est situé le C.L.S.C. La Source, et provenaient des données du recensement effectué en 1981 et disponibles au moment de notre commande statistique, en 1986. Ces seuils sont les suivants (CNBES, $1988: 11)$ :

$\begin{array}{lr}1 \text { personne } & 8045 \$ \\ 2 \text { personnes } & 10614 \\ 3 \text { personnes } & 14198 \\ 4 \text { personnes } & 16361 \\ 5 \text { personnes } & 19066 \\ 6 \text { personnes } & 20823 \\ 7 \text { pers. et }+ & 22919^{5}\end{array}$

Pour bâtir la carte de la pauvreté sur le territoire d'un C.L.S.C., il $s^{\prime}$ agit d'obtenir de Statistique Canada la proportion (\%) de ménages dont le revenu tombe en-dessous de leur seuil respectif, dans chaque plus petite unité de territoire pour laquelle les données du recensement sur le revenu par ménage sont disponibles. Cette unité territoriale est le secteur de dénombrement, plus petit que le secteur de recensement sur la base duquel les données sont habituellement publiées. À titre d'exemple, le territoire du C.L.S.C. La Source comptait 88 secteurs de dénombrement en 1981, alors que le nombre de secteurs de recensement n'était que de 16 .

Les données relatives à la proportion (\%) de ménages sous le seuil de pauvreté dans chaque secteur de dénombrement, non disponibles dans les publications de Statistique Canada, doivent faire l'objet d'une commande spéciale ${ }^{6}$. Notre commande, ainsi formulée "Ménages privés sous les seuils de pauvreté (tels que définis par I'utilisateur) par secteur de dénombrement, recensement de 1981 ", était accompagnée de la liste des numéros des secteurs de dénombrement sur le territoire du C.L.S.C. ${ }^{7}$ et de la liste des seuils de pauvreté par catégorie de ménage pour 1981.

Dans le traitement des données réalisé par Statistique Canada, l'ordinateur indique, pour chaque secteur de dénombrement, le 
nombre de ménages de une personne ayant un revenu inférieur à $8045 \$$, le nombre de ménages de deux personnes ayant un revenu inférieur à $10614 \$$, etc., pour arriver au total des ménages toutes catégories qui vivent sous le seuil de pauvreté. Dans notre cas, il était très important de ne commander que le total pour l'ensemble des ménages d'un secteur de dénombrement, et non par catégorie de ménages, parce que le programme de traitement des données de Statistique Canada est conçu de telle façon que les données concernant moins de 25 personnes, en 1981, n'apparaissent pas dans les compilations, pour des raisons de confidentialité. C'est un tiret (-) qui apparait ou encore l'expression "ce secteur est nul ", s'il s'agit d'un secteur de dénombrement totalisant moins de 25 ménages sous le seuil de pauvreté.

II arrive trop fréquemment que, dans un secteur de dénombrement, le nombre de ménages sous le seuil de pauvreté, dans une catégorie particulière de ménage, soit inférieur à 25 , pour qu'il soit utile de commander ce genre de compilation. Par ailleurs, il arrive rarement que le nombre total de ménages (toutes catégories) dans un secteur de dénombrement soit inférieur à 25 . Pour le territoire du C.L.S.C. La Source, par exemple, 20 secteurs de dénombrement présentaient cette caractéristique. À première vue, tout ce qu'on peut savoir à leur sujet est qu'ils comprennent moins de 25 ménages sous le seuil de pauvreté. Pour les fins de construction de la carte, ils devraient donc constituer une catégorie à part. Mais nous verrons plus loin qu'un artifice logique peut nous permettre d'en reclassifier une partie.

Pour tous les secteurs de dénombrement comprenant plus de 25 ménages sous le seuil de pauvreté, des nombres absolus pouvant être convertis en proportion (\%) sont disponibles, ce qui permet d'établir des catégories de secteur de dénombrement, selon la proportion de ménages vivant sous le seuil de pauvreté : les secteurs comprenant de 0 à $9,9 \%$ de ménages sous le seuil, les secteurs comprenant de 10 à $19,9 \%$, etc.

Parmi les secteurs de dénombrement comptant moins de 25 ménages sous le seuil de pauvreté, un certain nombre peuvent être reclassifiés dans la catégorie de 0 à $9,9 \%$, parce qu'en reportant le nombre " moins de 25 » sur le total des ménages du secteur, on arrive à moins de $9,9 \%$. Par exemple, "moins de 25 » sur 270 ménages donne moins de $9,3 \%$, donc un secteur qui entre de toute évidence dans la catégorie des secteurs comptant de 0 à $9,9 \%$ de ménages sous le seuil de pauvreté. Quand, par ailleurs, en reportant « moins de 25 » sur le total des ménages $d^{\prime}$ 'un secteur, on arrive à une proportion supérieure à $9,9 \%$, il n'est pas possible de reclassifier le secteur. Par exemple, " moins de 25 " sur 125 donne un résultat de " moins de 
$20 \%$ ", ce qui indique que le secteur peut appartenir à l'une ou l'autre des deux catégories suivantes : 0 à 9,9\% ou 10 à 19,9\%.

En reclassifiant tous les secteurs de « moins de 25 ménages sous le seuil de pauvreté " qui peuvent l'être, on arrive à la distribution suivante des secteurs de dénombrement du territoire du C.L.S.C. La Source : 10 secteurs de dénombrement sont sans résultat à cause des règles de confidentialité de Statistique Canada; 18 comptent de 0 à $9,9 \%$ de ménages sous le seuil de pauvreté; 28 comptent de 10 à 19,9\% de ménages sous le seuil; 20 comptent de 20 à 29,9\% de ménages sous le seuil; 12 comptent $30 \%$ et plus de ménages sous le seuil.

Pour cartographier ces résultats, il suffit de faire correspondre une couleur ou une trame à chacune des catégories mentionnées.

Dans l'étude du C.L.S.C. La Source, le choix a été fait d'approfondir la recherche sur les secteurs de dénombrement où la proportion (\%) de ménages sous le seuil de pauvreté est supérieure à celle de l'ensemble du territoire. Cette dernière était de $18,2 \%$ en 1981 . Les deux catégories de secteurs correspondant à cette définition sont ceux comptant de 20 à $29,9 \%$ de ménages sous le seuil et ceux comptant $30 \%$ et plus de ménages sous le seuil.

Les configurations que forment sur la carte les secteurs appartenant à ces deux catégories ont été délimitées et toutes les statistiques disponibles à leur sujet ont été analysées ${ }^{8}$. Huit zones de pauvreté ont ainsi été identifiées suivant les formes qu'elles évoquent : l'éventail, la botte, etc. Par exemple, l'éventail comprend les secteurs de dénombrement $069,107,108,109,110$ et 115, comme le montre l'illustration 1.

Pour chacune de ces zones et des secteurs qui les composent, les données suivantes ont été analysées : sexe, âge, activité selon le sexe et l'âge, niveau de scolarité selon le sexe, état matrimonial selon le sexe, proportion de locataires et de propriétaires, loyer brut, principales dépenses de propriété, état des logements, nombre de personnes par pièce, durée d'occupation, période de construction des logements. En somme, une série de données qui fournissent de précieuses indications pour orienter une intervention adaptée à ces zones prioritaires où les populations les plus "à risques" du territoire ont une très forte probabilité d'être concentrées.

\section{Des résultats qui lancent un défi}

Une étude comme celle-ci est susceptible d'interpeller les intervenants et intervenantes du C.L.S.C. L'image de banlieue à l'aise qui colle au territoire du C.L.S.C. La Source, à cause des nombreuses rues de résidences cossues de Charlesbourg, est sérieusement remise 


\section{ILLUSTRATION 1}

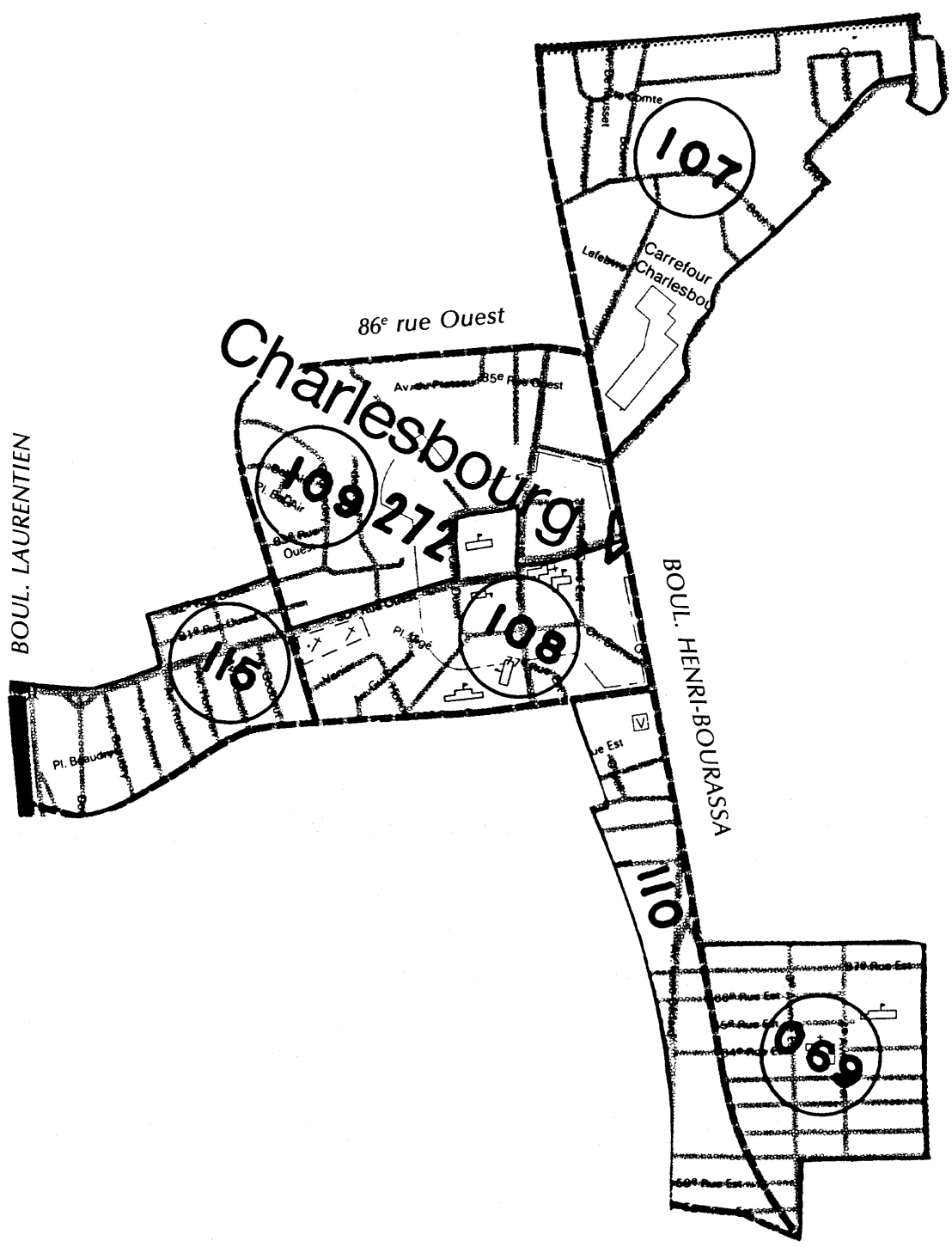

$68^{\mathrm{e}}$ rue Est 
en question. La proportion de ménages sous le seuil de pauvreté sur le territoire du C.L.S.C. La Source (18,2 \%) parle par elle-même. De plus, les zones de pauvreté ne sont pas confinées, comme on aurait pu s'y attendre, à ce qu'il est convenu d'appeler l' " arrière-pays » (la partie nord de Charlesbourg, Lac Saint-Charles et les Cantons-Unis de Stoneham et Tewkesbury). Elles s'insinuent au cœur même de la trame urbaine de Charlesbourg où est concentrée la majorité de la population du territoire.

Le portrait de la pauvreté sur le territoire invite à se donner un temps de réflexion pour s'outiller face à la réalité rendue manifeste par la recherche. En 1988 et 1989, des rencontres ont eu lieu avec les équipes du C.L.S.C., pour enrichir les résultats de la recherche des observations effectuées par les intervenants et intervenantes, dans le cours de leur pratique, et commencer à prospecter les moyens de mieux rejoindre la population des zones délimitées par l'enquête. Le réajustement des pratiques ne va pas de soi, dans un contexte où le temps de travail est déjà totalement canalisé dans les programmes institutionnalisés et les routines professionnelles établies. C'est pourtant sur des enjeux comme celui-ci que se joue la spécificité qu'on a voulu attribuer aux C.L.S.C., à leur origine, en tant qu'établissements liés aux besoins particuliers des communautés $d^{\prime}$ un territoire.

Le conseil d'administration du C.L.S.C. a été saisi des résultats de l'enquête et en a autorisé la diffusion publique, en encourageant une mise à jour avec les données du recensement de 1986. Le directeur général du C.L.S.C. a tenu une conférence de presse à l'intention non seulement des médias, mais aussi des dirigeants locaux.

L'idéologie néo-conservatrice dans laquelle nous baignons favorise le déni de réalité à l'égard de la pauvreté. Déjà, les changements survenus dans l'utilisation de l'espace sur le territoire (nouvelles constructions, mouvements de population) entre 1981 et 1986, sont invoqués, à l'occasion, pour minimiser la portée de l'étude, comme si on ne voyait pas les nouvelles concentrations de pauvreté qui, en même temps, se recréent ailleurs. Une mise à jour avec les données de 1986 répondra à cet argument. II restera toujours possible d'affirmer encore que la situation a bien changé entre 1986 et 1990, et qu'il faudra attendre le recensement de 1991. Heureusement, les rencontres effectuées jusqu'à maintenant nous laissent voir que plusieurs des intervenants, intervenantes et partenaires du C.L.S.C. ne recourent pas à cette fuite en avant et prennent acte du défi qui les confronte. 


\section{Notes}

* À la demande des auteurs, les génériques masculin et féminin sont utilisés dans I'article.

${ }^{1}$ Les auteurs remercient le directeur général du C.L.S.C. La Source, Louis Blanchette, de son intérêt pour la recherche dont il a facilité la réalisation et encouragé la publication.

${ }^{2} \mathrm{Au}$ chapitre des dépenses extraordinaires, il faut compter environ 6000 , si on engage un étudiant gradué pour exécuter les travaux de la recherche, et environ $2000 \$$ si la recherche est entièrement faite maison. Les dépenses courantes imputables à la recherche, minimes dans le premier cas, augmentent dans le deuxième en proportion du temps consacré à la recherche par le personnel régulier.

${ }^{3}$ Le ménage est défini par Statistique Canada comme " une personne ou groupe de personnes (autres que des résidents étrangers) occupant un même logement et n'ayant pas de lieu de résidence habituelle ailleurs au Canada. II se compose ordinairement d'un groupe familial, avec ou sans chambreurs, employés, etc. II peut aussi se composer de deux familles ou plus partageant le même logement, d'un groupe de personnes non apparentées ou d'une personne seule... " (Dictionnaire du recensement de 1981 : 78). La catégorie de ménages à laquelle s'applique notre distribution est celle des " ménages privés ", c'est-à-dire ceux regroupant des personnes qui occupent un "logement privé ". Sont exclus les "ménages collectifs", composés d'une personne ou d'un groupe de personnes occupant un " logement collectif ", c'est-à-dire un établissement commercial ou communautaire, tel que hôtel, motel, maison de chambres pour touristes, maison de repos, hôpital, résidence de personnel, caserne de camp militaire, camp de chantier, prison, centre d'accueil, pension et maison de chambres, etc. (Dictionnaire... : 78, 79, 88).

Nous avons constaté en cours de route qu'il aurait été plus approprié d'étudier la combinaison de deux distributions, les familles économiques et les personnes hors famille économique dans les ménages privés, plutôt que la distribution de ménages privés. La "famille économique " est un groupe de deux personnes ou plus qui vivent dans le même logement et qui sont apparentées par le sang, par alliance ou par adoption. Les personnes qui vivent en union libre sont considérées aux fins du recensement, comme actuellement mariées... " (Dictionnaire... : 65, 66; c'est nous qui mettons une partie du texte en relief). Cette option qui exige la commande de deux totalisations spéciales au lieu d'une seule, comme dans le cas des ménages privés, comporte par ailleurs l'avantage de compter comme des unités distinctes les personnes seules (chambreurs, employés, colocataires, etc.) qui partagent un logement avec une famille économique ou une ou plusieurs autres personnes seules, ou encore les familles économiques qui occupent un même logement.

Nous utiliserons cette double distribution dans la mise à jour de 1986 et vérifierons si des différences notables la démarquent de la distribution des ménages privés.

${ }^{4}$ Il s'agit de "familles économiques": voir plus haut, dans la note 3 . "Le revenu se compose des revenus en espèces reçus par tous les membres de la famille âgés de 15 ans et plus, et qui proviennent des sources suivantes : les salaires et traitements (avant déduction des impôts, de l'assurance-chômage, des pensions, etc.), le revenu net provenant d'un emploi autonome, les 
revenus de placements (le revenu d'intérêts, de dividendes, de loyers, etc.), les paiements de transferts gouvernementaux (tels que les allocations familiales, le crédit d'impôt pour enfants, les pensions de vieillesse, les crédits d'impôt provinciaux remboursables), les pensions (telles que les pensions de retraite et les rentes) et les revenus divers (tels que bourses d'études, pensions alimentaires). Ainsi les seuils de pauvreté se fondent sur le revenu brut plutôt que sur le revenu net (après l'impôt). " (CNBES, 1988 : 1-2).

${ }^{5}$ Une mise à jour avec les données du recensement de 1986 est présentement en cours. Les seuils correspondant à cette année sont les suivants (CNBES, $1988: 12)$ :

$\begin{array}{ll}1 \text { personne } & 10651 \$ \\ 2 \text { personnes } & 14053 \\ 3 \text { personnes } & 18799 \\ 4 \text { personnes } & 21663 \\ 5 \text { personnes } & 25243 \\ 6 \text { personnes } & 27571 \\ 7 \text { personnes et }+ & 30347\end{array}$

${ }^{6}$ La commande doit être adressée à Statistique Canada, Service aux utilisateurs, Édifice Jean-Talon, $2^{\mathrm{e}}$ étage, Ottawa, Ontario, K1A 0T7. Les ententes préliminaires peuvent être prises par téléphone au numéro (613) 951-3958. La personne-ressource avec qui nous transigeons pour la mise à jour de 1986 est monsieur Michel Séguin.

${ }^{7}$ La liste des secteurs de dénombrement correspondant aux municipalités qui composent le territoire du C.L.S.C. peut être trouvée dans les publications de Statistique Canada sur les divisions et subdivisions de recensement au Québec ( $\mathrm{n}^{\circ}$ 99-913 pour 1981 et 99-118 pour 1986).

Les fonds de cartes sur lesquels sont tracées les limites des secteurs de dénombrement peuvent être obtenus des organismes publics qui s'occupent d'aménagement du territoire; dans notre cas, le Service d'aménagement du territoire de la Communauté urbaine de Québec. On peut aussi s'adresser au bureau de Montréal de Statistique Canada auprès des personnes responsables : monsieur Guy Oddo (514-283-3316) ou monsieur Yvan Deslauriers (514-283-5742).

${ }^{8}$ Il s'agit de statistiques du recensement du Canada de 1981 qui ont été mises à notre disposition par le Bureau de la statistique du Québec (BSQ), 117, rue Saint-André, Québec, G1K 7W8, Tél.: 418-643-5116. Notre personne ressource dans cet organisme était madame Diane Haince. Cette partie de la recherche a été entièrement réalisée par Anne Racine qui a remis au C.L.S.C. un rapport de son travail : La pauvreté sur le territoire du C.L.S.C. La Source : une étude quantitative, juin 1987.

\section{Références bibliographiques}

Bellefeuille, R., "En milieux défavorisés. Le Québec a autant de naissances fragiles que dans le Tiers-Monde », Québec, Le Soleil, 9 février 1989 : A-1 et A-2.

Chamberland, C., "Famille, valeurs et société ", Service Social, vol. 36, n ${ }^{\text {os }}$ 2-3, $1987: 274-285$. 
CONSEIL NATIONAL DU BIEN-ÊTRE SOCIAL (CNBES), Les seuils de pauvreté de 1988 , Ottawa, CNBES, 1988.

Côté, C., G. Dussault, G. Doré, Les zones prioritaires de Québec, Rapport de recherche Ezop-Québec, Québec, COBEQ, 1971.

DUHAIME, R., "Le projet O.L.O. et la conscientisation. Une expérience terrain de périnatalité en milieu populaire ", dans AA. VV., Pratiques de conscientisation 2, Québec, Collectif québécois d'édition populaire, $1987: 215-245$.

Egbuonu, B.S. et B. Starfield, "Child health and social status », Pediatrics, vol. 69, n' 5, $1982: 550-557$.

ÉMOND, A. (sous la direction de), Et la santé, ça va ? Rapport de l'enquête Santé Québec 1987, tome 1, Québec, Les Publications du Québec, 1988.

Hollingshead, A.B. et F.C. Redlich, Social class and Mental Illness, New York, John Wiley, 1958.

LAPLANTE, P., " Rapport-synthèse " dans Opération : rénovation sociale, Montréal, Conseil des œuvres, $1966: 16,32-33$.

MAYER-RENAUD, M., "Le statut socio-économique : construction d'un indice utile pour la gestion sociale ", Sociologie et sociétés, vol. Xx, $n^{\circ} 1,1988$ : 47-59.

Nersesian, W.S., M.R. Petit, R. Shaper, D. Lemieux et E. NaOr, "Childhood death and poverty : A study of all childhood deaths in Maine, 1976 to 1980 ", Pediatrics, vol. 75, no 1, 1985 : 41-50.

SCHLESINGER, B., "La pauvreté dans une société opulente", Service social, vol. 14, $n^{\circ} 1,1965: 4-15$. 